\title{
Bushenhuoxue Formula Facilitates Articular Cartilage Repair and Attenuates Matrix Degradation by Activation of TGF- $\beta$ Signaling Pathway
}

\author{
Rui Dong $\mathbb{D}^{1},{ }^{1,2}$ Jun Ying $\left(\mathbb{D},{ }^{1,2}\right.$ Taotao Xu ${ }^{1},{ }^{3}$ Songfeng Hu ${ }^{1 D},{ }^{4}$ Peng Zhang, ${ }^{1,2}$

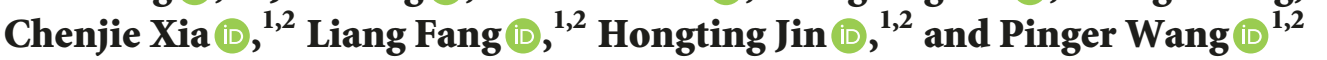 \\ ${ }^{1}$ The First Clinical Medical College of Zhejiang Chinese Medical University, Hangzhou 310053, Zhejiang, China \\ ${ }^{2}$ Institute of Orthopaedic and Traumatology of Zhejiang Province, Hangzhou 310053, Zhejiang, China \\ ${ }^{3}$ Department of Orthopaedic Surgery, The First Affiliated Hospital of Zhejiang Chinese Medical University, \\ Hangzhou 310006, Zhejiang, China \\ ${ }^{4}$ Department of Orthopaedics, Shaoxing Hospital of Traditional Chinese Medicine, Shaoxing 312000, Zhejiang, China
}

Correspondence should be addressed to Hongting Jin; hongtingjin@163.com and Pinger Wang; apple63209321@126.com

Received 12 August 2018; Accepted 23 September 2018; Published 8 October 2018

Academic Editor: Yoshiki Mukudai

Copyright (C) 2018 Rui Dong et al. This is an open access article distributed under the Creative Commons Attribution License, which permits unrestricted use, distribution, and reproduction in any medium, provided the original work is properly cited.

\begin{abstract}
Objective. To investigate the effect and underlying mechanism of Bushenhuoxue (BSHX) formula on articular cartilage repair. Methods. Twenty-four full-thickness cartilage defect rats were divided into two groups: model group and BSHX group (treated with BSHX formula). Macroscopic observation and histopathological study were conducted after 4- and 8-week treatment. Additionally, we also evaluated chondrocyte proliferation, extracellular matrix (ECM) deposition, cartilage degradation, and chondrocyte hypertrophy-related genes expression in chondrogenic ATDC5 cells cultured in BSHX formula-mediated serum. Moreover, we assessed aforementioned genes expression and pSMAD2/3 protein level in Tgf $\beta \mathrm{r} 2$ siRNA transfected chondrogenic ATDC5 cells in order to address whether BSHX formula exerts cartilage repairing effect through TGF- $\beta$ signaling. Results. Neocartilage regeneration promotion effect was observed in cartilage defect rats after BSHX formula treatment, with increases in Col 2 and pSMAD2 and decreases in Mmp13 and Runx2. Moreover, cell proliferation, the elevated Col2al, Aggrecan and pSMAD2/3, reduced Mmp13, Adamts5, Col10al, and Runx2 expression were also observed in chondrogenic ATDC5 cells cultured in BSHX formulamediated serum. Besides, the expression alteration of ECM deposition, cartilage degradation, chondrocyte hypertrophy-related genes, and pSMAD2/3 protein levels presented in Tgf $\beta \mathrm{r} 2$ downregulated chondrogenic ATDC5 cells couldn't be adjusted by BSHX formula treatment. Conclusion. By activation of TGF- $\beta$ signaling, BSHX formula can promote articular cartilage repair by accelerating chondrocyte proliferation and maintaining chondrocyte phenotype, upregulate ECM accumulation, and inhibit matrix degradation.
\end{abstract}

\section{Introduction}

Articular cartilage is a highly specialized tissue; its unique feature owns just one cell type: the chondrocyte, which exhibits low proliferation activity and is imbedded in abundant extracellular matrix (ECM) [1]. Furthermore, this distinctive tissue plays an important role in joint movement, since it acts as a cushion in bone articulation which absorbs mechanical loading and also produces lubricating fluid to decrease joint friction. This built-for-life tissue exhibits poorly self-healing capacity in adulthood due to its nonvascular and noninnervated properties [2, 3]. However, repetitive mechanical loading and many pathologic factors can cause cartilage damage in the general and athletic populations $[4,5]$. The cartilage lesion patients usually suffered from pain, swelling, and joint locking [6], which eventually lead to progressive damage and degenerative diseases, namely, osteoarthritis (OA) which is the leading cause of physical disability [7]. 
Numerous techniques are commonly used for cartilage repair. Microfracture acts as the most widely used marrow stimulation technique which could facilitate tissue regeneration and provide immediate relief of symptoms [8], but decreasing physical function after early improvement and increasing failure rate indicate the long-term clinical limitations of this operation [9]. Mosaicplasty such as autologous osteochondral transplantation and osteochondral allografting has reported some positive results but also presents disadvantages like procedure complexity, lack of integration, and instability [10-12]. Recently, tissue engineering has become an acceptable way for treating cartilage defect; although it may partly overcome these drawbacks, the optimal selection of seed cells and scaffold has not yet been well elucidated [13]. Moreover, inadequate biomechanical property often causes degradation of the implant. Generally speaking, none has so far been approved a promising way for cartilage regeneration.

Traditional Chinese medicine (TCM), which was applied for treating many disorders for thousands of years, has become a promising complementary and alternative medicine for osteoarthritis with limited side effects. It also shows strong effects in articular cartilage protection [14,15]. According to the TCM theory, homeostasis maintenance of bone and cartilage and its regeneration rely on sufficient essence of kidney and liver [16]. Deficiency of kidney and liver combined with blood stasis can eventually cause OA-related cartilage damage. Bushenhuoxue (BSHX) formula, a tonifying kidney and promoting blood circulation decoction, has been clinically used for many years in OA treatment and yield positive outcome $[17,18]$. Besides, we previously testified BSHX formula which inhibits cartilage degradation in OA mouse model [19], but it is not clear whether it can promote articular cartilage repair.

The transforming growth factor beta (TGF- $\beta$ ) signaling plays a vital role in cartilage homeostasis. Latent TGF- $\beta$ binding to type II receptor ( $\operatorname{Tfg} \beta \mathrm{r} 2$ ) leads to recruitment and activation of type I receptor, which could phosphorylate its downstream target mothers against decapentaplegic homolog 2 (Smad2) and Smad3 finally regulating the expression of target genes such as collagen II ( $\mathrm{Col} 2)$, matrix metallopeptidase 13 (Mmp13), and Runt-related transcription factor 2 (Runx2). In mammalian cells, activation of TGF- $\beta$ signaling regulates cell differentiation, promotes chondrocytes proliferation, and improves aggregation of $\mathrm{Col} 2$ and proteoglycan, preventing it from hypertrophy [20]. Therefore, pharmacological activation of TGF- $\beta$ signaling has been proposed to accelerate articular cartilage repair $[21,22]$.

The purpose of the current study was the histological observation of the cartilage repair promoting effect of BSHX formula on rat cartilage defect model. We also testify this positive effect of BSHX formula by incubating chondrogenic ATDC5 cells in BSHX formula-mediated serum. For potential mechanism elucidation, we downregulate TGF- $\beta$ signaling in chondrogenic ATDC5 cells by $T g f \beta r 2$ siRNA to verify whether BSHX formula exerts articular cartilage repairing effect through this pathway.

\section{Materials and Methods}

2.1. Preparation of BSHX Formula. Ten herbal medicines in BSHX formula (Table 1) were provided by the First Affiliated
Hospital of Zhejiang Chinese Medical University (Hangzhou, China). The exact chemical constituents and extraction process were described as follows. Briefly, eight abovementioned herb medicines were mixed in a ratio of 3:2:2:2:1:1:2:1 for aqueous extraction: Rehmannia glutinous (Liboscb), Eucommia ulmoides (Oliv.), Aconitum carmichaeli (Debx.), Lycium barbarum (L.), Cornus officinalis (Sieb.), Carthamus tinctorius (L.), Dioscoreae opposite (Thunb.), and Glycyrrhiza uralensis (Fisch.). Ethanol extraction was used for Cinnamomum cassia (Presl) and Prunus persica (Batsch.) in a ratio of 1:2. Finally, two types of extracts were mixed and concentrated to $2 \mathrm{~g}$ crude drug/ml for further use.

The analysis and quality control of BSHX were identified by the high-performance liquid chromatography assay (HPLC). Six of the major constituents including loganin, amygdalin, pinoresinol diglucoside, liquiritin, cinnamaldehyde, and hydroxysafflor yellow A were identified in our previous study [19].

2.2. Animals and Modeling. Twenty-four Sprague-Dawley rats (male, 12 -week-old) were anesthetized by intraperitoneal injection of $1 \%$ pentobarbital sodium solution $(0.3 \mathrm{ml} / 100 \mathrm{~g})$. The knee joints were opened through a medial parapatellar incision, and femoral trochlea was exposed directly after patella dislocation. Electric drill was used for drilling a full thickness cartilage defect on the surface of trochlear groove ( $2 \mathrm{~mm}$ diameter $2 \mathrm{~mm}$ depth), and incision was then sutured after patella dislocation was reduced. After surgery, all rats were treated with penicillin intramuscularly injected at $8 \mathrm{U} /$ rat for 3 consecutive days. All studies were approved by the Committee on the Ethics of Animal Experiments of Zhejiang Chinese Medical University.

2.3. Grouping and Drug Administration. All rats were divided into two groups randomly ( $n=12 /$ group at $4 \mathrm{w}$ and $8 \mathrm{w})$ : model group and BSHX group. The day after surgery, BSHX formula was orally administered to BSHX group once a day at a dose of $0.7 \mathrm{ml} / 100 \mathrm{~g}$ body weight. The exact dose of oral administration was determined according to dosage conversion formula [23]. The same dose of $0.9 \%$ normal saline was fed to model group. All rats were sacrificed at $4 \mathrm{w}$ and $8 \mathrm{w}$; the knee joint specimens were collected for further analysis.

2.4. Gross Conference and Histological Analysis. Knee joints were washed in $1 \times$ phosphate buffer saline (PBS) and photographs were then being captured in order to evaluate the status of cartilage by using the International Cartilage Repair Society (ICRS) cartilage repair assessment [24]. In this scoring system, lower score represents worse cartilage.

For histological analysis, specimens were fixed in $4 \%$ paraformaldehyde for $72 \mathrm{~h}$, decalcified with $14 \%$ EDTA solution $(\mathrm{PH}=7.4)$ for about $30 \mathrm{~d}$, then dehydrated and embeded in paraffin. $3 \mu \mathrm{m}$ sagittal sections were stained with Alcian Blue Hematoxylin/Orange $\mathrm{G}(\mathrm{ABH})$ for gross structural analyzing. Mankin scoring system which graded cartilage on a scale of 0 13 was used to evaluate the cartilage lesion [25]. Higher score means worse repairing. Either ICRS assessment or Mankin scoring system was evaluated by two independent observers. 
TABLE 1: The compositions of Bushenhuoxue (BSHX) formula.

\begin{tabular}{|c|c|c|c|c|}
\hline Chinese name & Botanical name & Latin name & Parts used & Proportion \\
\hline Shu Di Huang & Rehmannia glutinosa(Liboscb) & Rehmannia glutinous (Liboscb) & Root & $17.4 \%$ \\
\hline Du Zhong & Eucommia ulmoides (Oliv.) & Eucommiae cortex & Bark & $11.8 \%$ \\
\hline $\mathrm{Fu} \mathrm{Zi}$ & Aconitum carmichaeli (Debx.) & Aconitilateralis radix preparata & Root & $11.8 \%$ \\
\hline Gou Qi & Lycium barbarum (L.) & Lyciifructus & Fruit & $11.8 \%$ \\
\hline Rou Gui & Cinnamomum cassia (Presl) & Cinnamomi cortex & Bark & $5.9 \%$ \\
\hline Shan Zhu Yu & Cornus officinalis (Sieb.) & Cornifructus & Fruit & $5.9 \%$ \\
\hline Tao Ren & Prunus persica (Batsch.) & Semen Persicae & Fruit & $11.8 \%$ \\
\hline Hong Hua & Carthamus tinctorius (L.) & Carthamus tinctorius (L.) & Corolla & $5.9 \%$ \\
\hline Shan Yao & Dioscoreae opposite (Thunb.) & Dioscoreae Rhizoma & Root & $11.8 \%$ \\
\hline Gan Cao & Glycyrrhiza uralensis (Fisch.) & Glycyrrhizae Radix et Rhizoma & Root & $5.9 \%$ \\
\hline
\end{tabular}

2.5. Immunohistochemistry (IHC) Analysis. After deparaffination and rehydration, $3 \mu \mathrm{m}$ sagittal sections were treated as follows: $0.3 \%$ hydrogen peroxide for $10 \mathrm{~min}$ to reduce endogenous peroxidase activity and $0.3 \%$ triton $\mathrm{x}-100$ for increasing cell membrane permeability if necessary. Sections were then incubated in $0.1 \mathrm{~mol} / \mathrm{L}$ citrate buffer $(\mathrm{PH}=4.0)$ in $60^{\circ} \mathrm{C}$ for $4 \mathrm{~h}$ antigen retrieval. $5 \%$ normal goat serum (Invitrogen, MD, USA) was used to block nonspecific staining for $20 \mathrm{~min}$ at room temperature. Sections were treated with anti-Col 2 (diluted 1:1000, Millipore, mab1330, USA), antiRunx2 (diluted 1:200, abcam, ab76956, UK), anti-Mmp13 (diluted 1:200, abcam, ab39012, UK), and anti-pSMAD2 (diluted 1:100, abcam, ab188334, UK) primary antibody and incubated overnight at $4^{\circ} \mathrm{C}$. Second antibody goat antimouse/rabbit antibody (diluted 1:1000) was added for $20 \mathrm{~min}$, and diaminobenzidine solution (Invitrogen, MD, USA) was used for detecting positive staining while hematoxylin for counterstaining.

2.6. Preparation of BSHX Formula-Mediated Serum. Twenty Sprague-Dawley rats (8-week-old, weigh at $200 \pm 20 \mathrm{~g}$ ) were randomly divided into BSHX group and control group. The rats in BSHX group underwent oral administration of BSHX ( $1 \mathrm{mg} / 10 \mathrm{~g}$ body weight/day) once a day for 7 days; meanwhile, the model group received $0.9 \%$ normal saline at the same dose. The blood samples were collected separately from the abdominal aorta; after being centrifuged at $3000 \mathrm{rpm} / \mathrm{min}$ at $4^{\circ} \mathrm{C}$ for $10 \mathrm{~min}$, the serum of both groups was isolated and stocked at $-80^{\circ} \mathrm{C}$ until required.

2.7. Cell Culture. ATDC5 cells (Riken Cell Bank, Ibaraki, Japan) underwent chondrogenesis by ITS (insulin, transferrin and selenous acid) (Gibco BRL, MD, USA) and were cultured in Dulbecco's modified Eagle's medium/Ham's F-12 medium (DMEM/F12; 1:1 mixture) which contained 10\%Fetal bovine serum plus $50 \mathrm{U} / \mathrm{ml}$ penicillin and $50 \mathrm{mg} / \mathrm{ml}$ streptomycin for two weeks, incubated in $37^{\circ} \mathrm{C}, 5 \% \mathrm{CO}_{2}$ humidified atmosphere.

2.8. Cell Proliferation Assay. Cell proliferation was tested by Cell Counting Kit-8 (CCK-8, Dojindo Laboratories, Kumamoto, Japan) following manufacturer's protocol. $100 \mu \mathrm{l}$ of chondrogenic ATDC5 cells suspensions $\left(2 \times 10^{3}\right.$ cells/well $)$ were added into 96-well plates overnight. After the supernatant was removed, cells were then incubated in FBS-free medium which contains different concentrations of BSHX formula-mediated serum for $24 \mathrm{~h}$. $10 \mu \mathrm{l} \mathrm{CCK} 8$ solution was added to each well incubation for $3 \mathrm{~h}$ and the optimal concentration of BSHX formula-mediated serum was measured at $450 \mathrm{~nm}$.

2.9. Transfection. Chondrogenic ATDC5 cells were transfected with Tgf $\beta \mathrm{r} 2$ small interfering RNA (Tgf $\beta \mathrm{r} 2$ siRNA, RiboBio, Guangzhou, China) by Lipofectamine RNAiMAX Transfection Reagent (Invitrogen, USA). The sequence of Tgf $\beta$ r 2 siRNA was $5^{\prime}$-CCUGUUGCCUGUGUGACUU-3' (sense) and $3^{\prime}$-GGACAACGGACACACUGAA-5' (antisense). The day before transfection, cells $\left(1 \times 10^{5}\right.$ cells/well $)$ were plated into 6-well plates overnight and then incubated with transfection mixtures containing Tgf $\beta \mathrm{r} 2$ siRNA (final concentration $20 \mathrm{nM}$ ) or negative control siRNA (final concentration $20 \mathrm{nM}$ ) for $6 \mathrm{~h}$. After transfection, the mixtures were replaced with complete culture medium. Transfection efficiency was measured at $24 \mathrm{~h}$ and $72 \mathrm{~h}$ by the expression level of Tgf $\beta \mathrm{r} 2$ mRNA through fluorescence quantitative PCR.

2.10. Quantitative Gene Expression Analysis. Total RNA was extracted from cells by using TRIzol (Invitrogen, CA, USA), isolated RNA was then reverse transcribed to cDNA using RevertAid First Strand cDNA Synthesis Kit (Invitrogen, CA, USA) according to manufacturer's procedure, fluorescence quantitative PCR was conducted by SYBR ${ }^{\circledR}$ Premix Ex Taq ${ }^{\text {TM }}$ II (Takara, China), and the relative primers are present in (Table 2). The expression level of mRNAs was normalized to $\beta$-actin, respectively, as endogenous control and was calculated using the $2^{-\Delta \Delta \mathrm{Ct}}$ method.

2.11. Western Blot Analysis. Cells were divided into four groups as follows: negative control siRNA (NC siRNA), NC siRNA + BSHX, Tgf $\beta r 2$ siRNA, and Tgf $\beta$ r 2 siRNA + BSHX. All cells were lysed in lysis buffer to obtain total protein extracts, respectively. After being incubated on ice for $30 \mathrm{~min}$, the extracts were separated with 12\% SDS-PAGE gel and 
TABLE 2: Primer name and sequences for PCR analysis.

\begin{tabular}{|c|c|}
\hline Primer name & Sequence \\
\hline$\beta$-actin forward & $5^{\prime}$-GGAGATTACTGCCCTGGCTCCTA-3' \\
\hline$\beta$-actin reverse & 5'-GACTCATCTACTCCTGCTTGCTG-3' \\
\hline Col2al forward & $5^{\prime}$-TGGTCCTCT GGGCATCTCAGGC-3' \\
\hline Col2al reverse & $5^{\prime}$-GGTGAACCTGCTGTTGCCCTCA-3' \\
\hline Mmp13 forward & $5^{\prime}$-TTTGAGAACACGGGGAAGA-3' \\
\hline Mmp13 reverse & 5'-ACTTTGTTGCCAATTCCAGG-3' \\
\hline Aggrecan forward & $5^{\prime}$-CGCCACTTTCATGACCGAGA-3' \\
\hline Aggrecan reverse & $5^{\prime}$-TCATTCAGACCGATCCACTGGTAG-3' \\
\hline Adamts 5 forward & $5^{\prime}$-CCAAATGCACTTCAGCCACGATCA-3' \\
\hline Adamts5 reverse & $5^{\prime}$-AATGTCAAGTTGCACTGCTGGGTG-3' \\
\hline Col10al forward & $5^{\prime}$-ACCCCAAGGACCTAAAGGAA- $3^{\prime}$ \\
\hline Col10a1 reverse & $5^{\prime}$-CCCCAGGATACCCTGTTTTT-3' \\
\hline Runx2/Cbfal forward & $5^{\prime}$-GAGGGCACAAGTTCTATCTGGA-3' \\
\hline Runx2/Cbfal reverse & $5^{\prime}$-GGTGGTCCGCGATGATCTC-3' \\
\hline
\end{tabular}

transferred to PVDF membranes. The membranes were then blocked in 5\% milk for $1 \mathrm{~h}$ and incubated with different primary antibodies against phosphorylated-SMAD2/3 (diluted 1:1000, Cell Signaling Technology, \#8828, USA) and SMAD2/3 (diluted 1:1000, Cell Signaling Technology, \#8685, USA) overnight at $4^{\circ} \mathrm{C}$. Next day, the membranes were incubated with goat anti-rabbit horseradish peroxidaseconjugated secondary antibody (diluted 1:5000; Abcam, ab6721, USA) at room temperature for $1 \mathrm{~h}$. The densities of the bands were visualized by chemiluminescence.

2.12. Statistical Analysis. All data were described as mean \pm standard deviation. Independent-samples t-test and oneway ANOVA test which were used in the present research were performed using SPSS software (version24.0, IBM Corporation, Armonk, NY, USA), and statistically significant changes were classified as significant $(*)$ while $p<0.05$ and highly significant (\#) while $P<0.01$.

\section{Results}

3.1. BSHX Improves the Gross Appearance and Accelerates Tissue Regeneration of Cartilage Defects. As shown in Figure 1(a), all specimens showed different degrees of cartilage damage. At 4 weeks, the defect area in the model group repaired slightly as it presented obvious lacuna with less regenerated tissue; meanwhile, plenty of regenerated tissue appeared in defect site of BSHX group but the surface was still rugged and lower than surrounding tissue. At 8 weeks, most parts of defect were filled by regenerated tissue covered with fibrillated surface. In model group, the surface of defects seemed rugged with big scratch on the junction between the defect sites and normal area. In BSHX group, the defected surface presented more smoothly and the regenerated tissue appeared similar to the native cartilage. The result of ICRS cartilage repair score was consistent with gross observation, and the macroscopic score of model group was higher than BSHX group in either $4 \mathrm{w}$ or $8 \mathrm{w}(P<0.01)$ (Figure $1(\mathrm{~b}))$.
To figure out the cellular organization and ECM deposition of both groups, we then performed $\mathrm{ABH}$ staining (Figure 1(c)). In model group at $4 \mathrm{w}$, the defects presented obvious lacuna which caved in new tissue containing thin fibrocartilage and plenty fibroblast morphologic cells. The defects were filled with new tissue over $50 \%$ depth at $8 \mathrm{w}$; hardly any hyaline-like cartilage could be found in the regenerated surface. In BSHX group, the defects exhibited about $75 \%$ repair of the defect depth at $4 \mathrm{w}$, the regenerated tissues were comprised of fibrocartilage and fibroblast morphologic cells. Astonishingly, the repaired tissue exhibited a slice of hyaline-like cartilage morphology and few glycosaminoglycans accumulations at $8 \mathrm{w}$. BSHX group had lower Mankin score than model group in $4 \mathrm{w}$ and $8 \mathrm{w}(P<0.01)$ (Figure $1(\mathrm{~d}))$. Altogether, BSHX group presents better cartilage morphology and higher surface regularity than model group.

\subsection{BSHX Maintains the Homeostasis of Articular Cartilage} and Chondrocytes. To explain the changes identified by histology, we performed IHC on femur condylar of both groups. IHC for Col2 illustrated the expression of $\mathrm{Col} 2$ in newly formed tissue and surrounding normal cartilage was lower at both times in model group than BSHX group $(P<0.01)$, and the regenerated tissue in BSHX group seemed almost similar to the surrounding cartilage (Figures 2(a) and 2(b)). We also performed IHC for MMP13, a key catabolic enzyme which took responsibility for cartilage damage and degradation. As expected, BSHX group showed lower expression in both $4 \mathrm{w}$ and $8 \mathrm{w}$ compared to model group $(P<0.05)$ (Figures $2(\mathrm{c})$ and $2(\mathrm{~d}))$. In addition, we detected a significant decrease in expression of Runx2 in BSHX group at $4 \mathrm{w}(P<0.05)$ and $8 \mathrm{w}$ $(P<0.01)$ (Figures $2(\mathrm{e})$ and $2(\mathrm{f})$ ), which marks chondrocytes hypertrophy.

To extend our in vivo study, we then treated chondrogenic ATDC5 cells with BSHX formula containing culture medium. CCK-8 assay was performed for optimal serum concentration selection and cell proliferation assessment. Results showed obviously positive dosage-effect relation between 


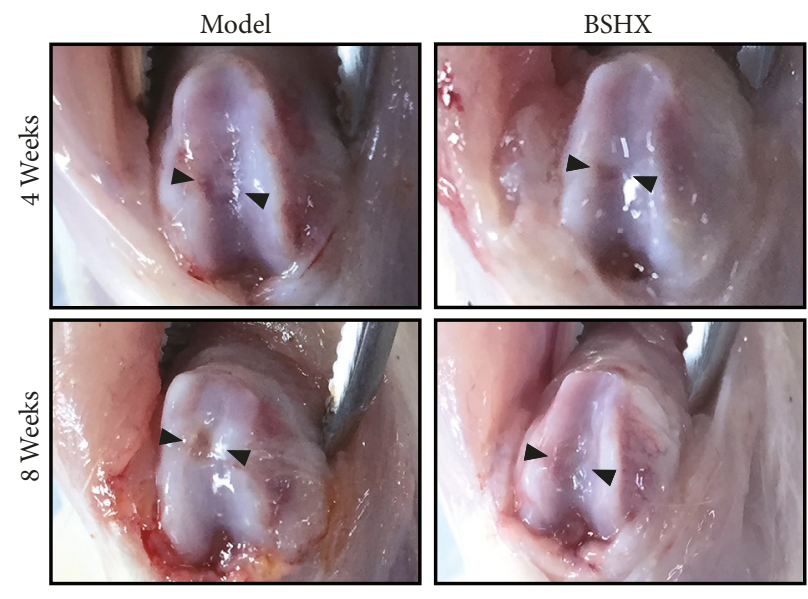

(a)

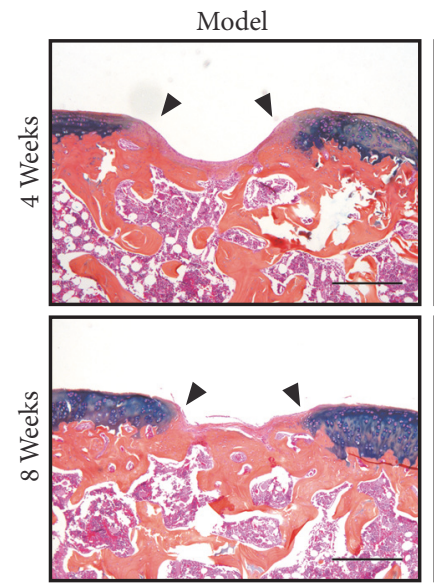

(c)

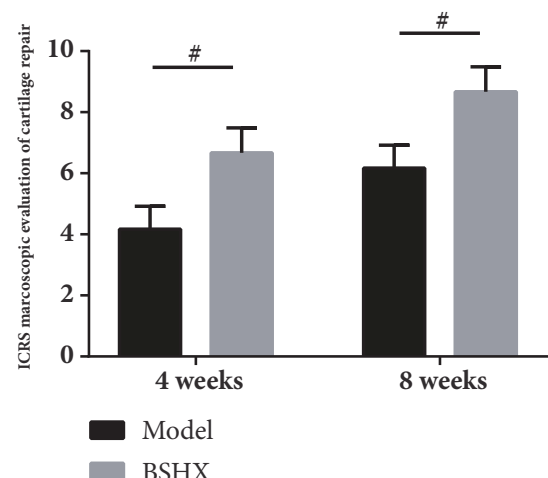

(b)

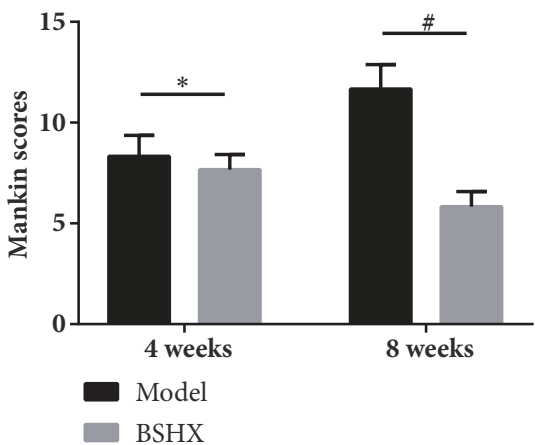

(d)

FIGURE 1: (a) Gross appearance of both model group and BSHX group at 4 and 8 weeks. (b) Assessment of ICRS cartilage repair score. (c) $\mathrm{ABH}$ staining for histological sections of both groups. Bar $=50 \mu \mathrm{m}$. (d) Mankin score for cartilage repair. $* P<0.05, \# P<0.01$.

cell proliferation and the concentration of serum; meanwhile, 10\% BSHX-serum was the most efficient concentration (Figure 3(a)).

Fluorescence quantitative PCR was performed on RNA isolated from the cells of BSXH group and control group at $48 \mathrm{~h}$. We first detected a significant elevation in the expression of Col2al and Aggrecan (Figure 3(b)), which presents ECM deposition. Next, we examined genes associated with cartilage degradation and saw a significant decrease in the level of Mmp13 and Adamts5 (Figure 3(c)). In addition, we looked at genes that define chondrocyte hypertrophy and terminal differentiation; we found that Colloal and Runx2 were all suppressed in BSHX group (Figure 3(d)). We believe BSHX formula can promote chondrocyte proliferation, maintain its characteristics, and prevent cartilage degeneration.

3.3. BSHX Exerts Its Articular Cartilage Repairing Effect through TGF- $\beta$ Signaling Pathway. Next, we wanted to determine if BSHX formula exerts the abovementioned cartilage repairing effect through TGF- $\beta$ pathway. We first performed IHC for pSMAD2 in both groups at $4 \mathrm{w}$; as expected, BSHX group presented more pSMAD2-positive cells than model group $(\mathrm{P}<0.01)$ (Figures $4(\mathrm{a})$ and $4(\mathrm{~b})$ ), which indicated the activation of TGF- $\beta$ pathway.

To further explain this phenomenon, we then discussed it in vitro. The concentration of $20 \mathrm{nM} \operatorname{Tgf} \beta \mathrm{r} 2$ siRNA was transfected into chondrogenic ATDC5 cells, and no-silence siRNA group as negative control. We assessed the expression of Tgf $\beta \mathrm{r} 2$ in these cells. We found the level of Tgf $\beta \mathrm{r} 2 \mathrm{mRNA}$ has been decreased about $70 \%$ of control values in $24 \mathrm{~h}$ and $72 \mathrm{~h}$, whereas there was no significant difference between $24 \mathrm{~h}$ and $72 \mathrm{~h}$ in transfection efficiency (Figure 4(c)).

After transfection, the cells were divided into three groups: Tgf $\beta \mathrm{r} 2$ siRNA group and negative control siRNA group (NC siRNA) were treated with complete culture medium containing BSHX-free rat serum whereas Tgf $\beta$ r2 siRNA plus BSHX group were treated with complete culture medium containing $10 \%$ BSXH formula-mediated serum; all groups were incubated at related culture medium for $48 \mathrm{~h}$. As a result, compared to NC siRNA group, Tgf $\beta$ r2 prominently decreased the expression of Col2al and Aggrecan, but BSHX formula could not reverse this tendency (Figure 4(d)). 


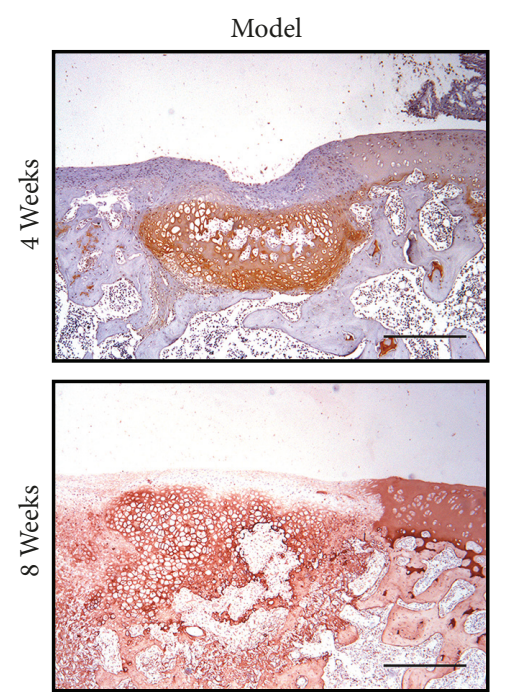

(a)
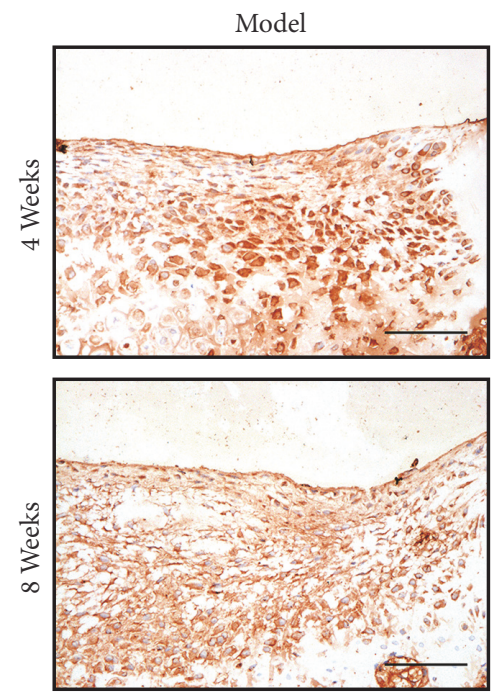

(c)
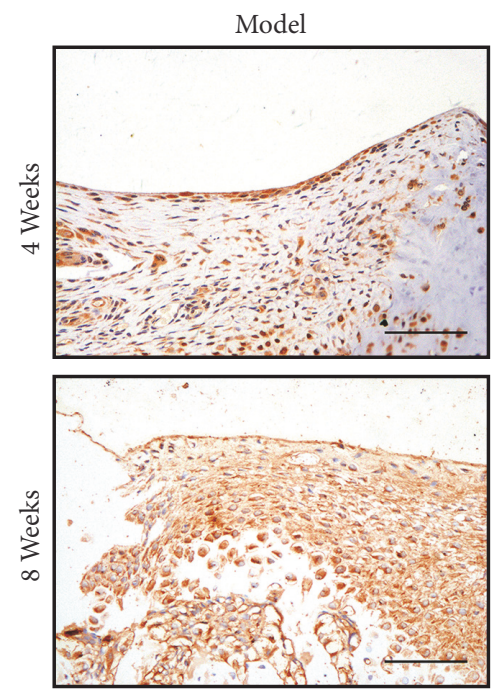

(e)

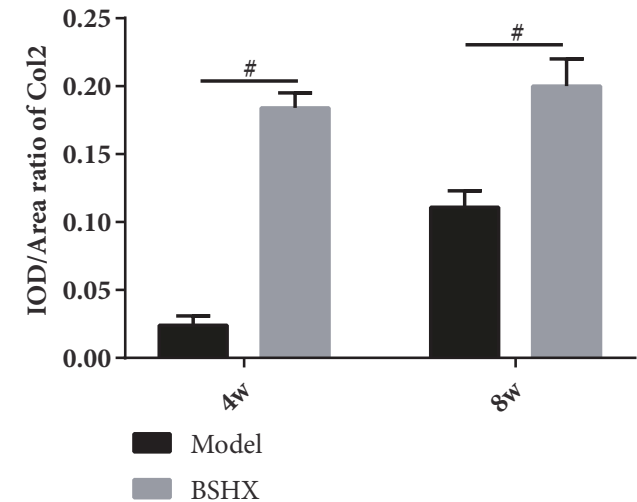

(b)

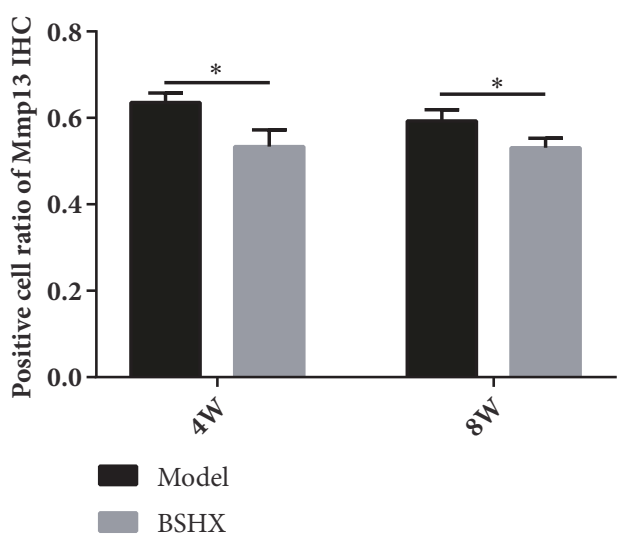

(d)
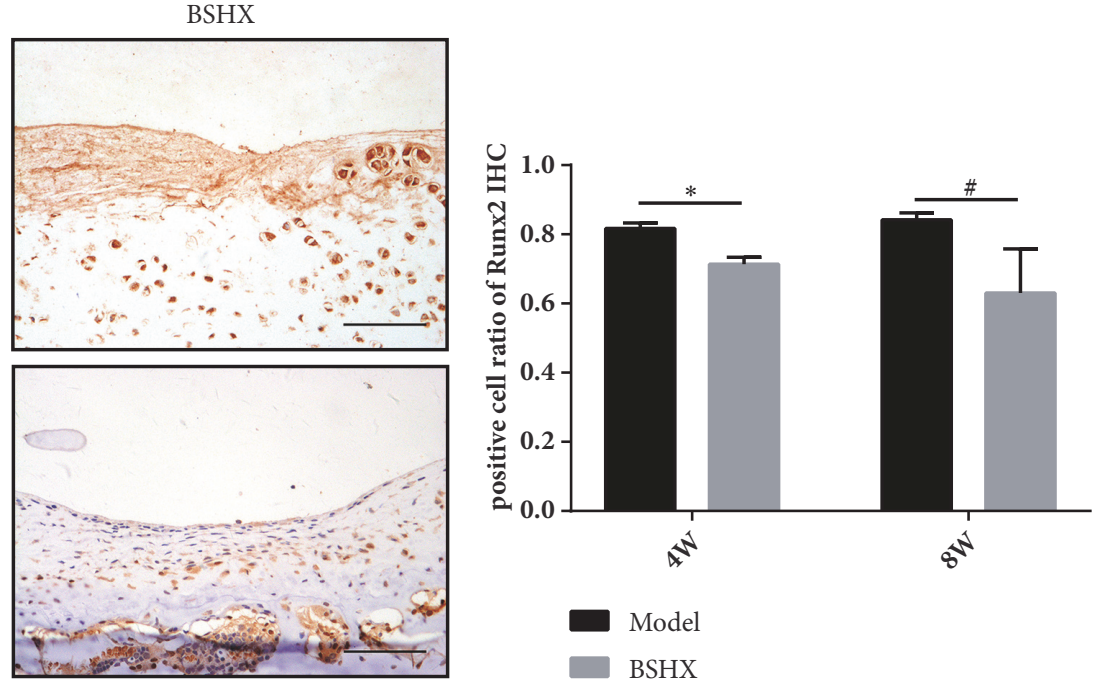

(f)

FIGURE 2: (a) Immunohistochemistry for Col II in both groups at 4 and 8 weeks, Bar=50 $\mu \mathrm{m}$. (b) IOD/Area ratio of Col2 expression. (c) IHC for Mmp13, Bar=200 $\mu \mathrm{m}$. (d) Positive cell ratio of Mmp13. (e) IHC for Runx2, Bar=200 $\mu \mathrm{m}$. (f) Positive cell ratio of Runx2. $* P<0.05$, \#P<0.01. 

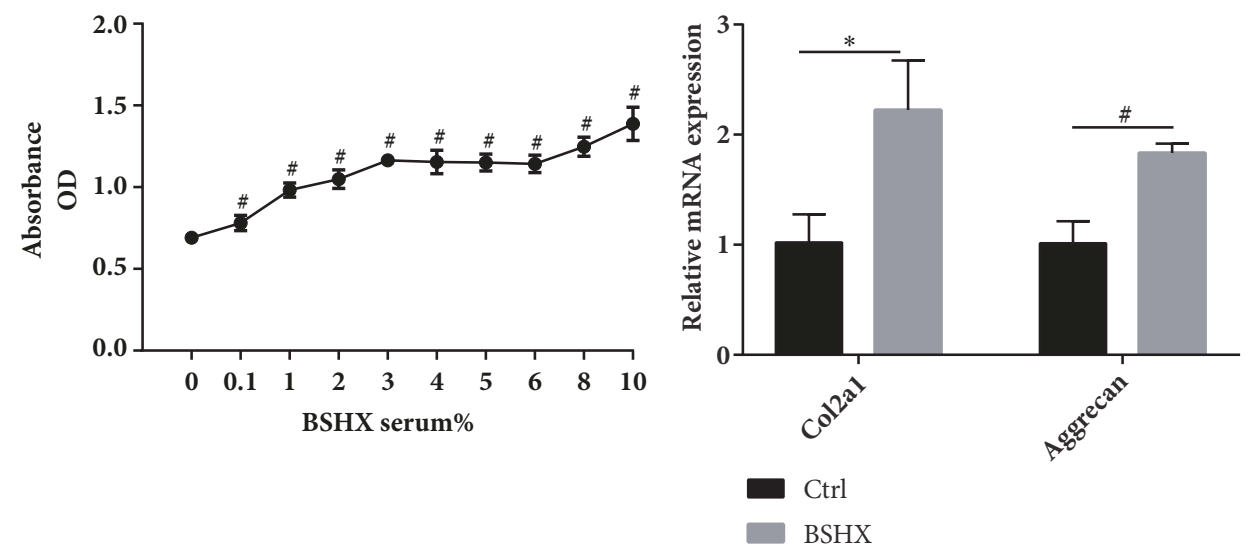

(a)

(b)
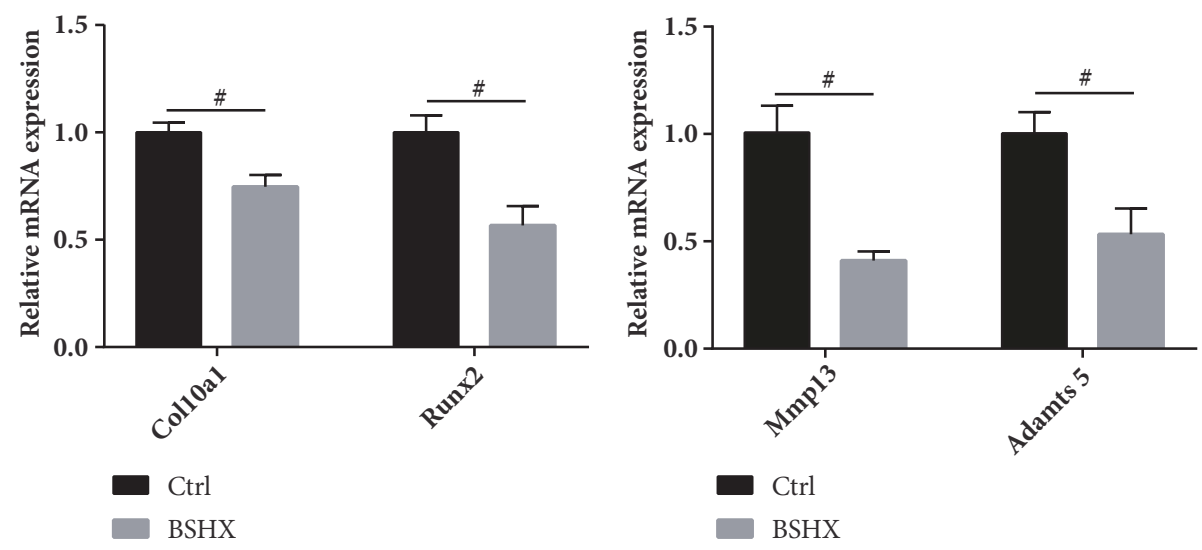

(c)

(d)

FIGURE 3: (a) Absorbance values of CCK8 test for cell proliferation and optimal BSHX-mediated serum concentration selection in chondrogenic ATDC5 cells after BSHX formula treatment. The expression alterations in Col2al and Aggrecan mRNA (b), Mmp13 and Adamts5 mRNA (c), Col10al and Runx2 mRNA (d). $* P<0.05$, $\# P<0.01$.

Similarly, the expression of Mmp13, Adamts5, Col10al, and Runx2 was dramatically upregulated by partial blockage of TGF- $\beta$ signaling while BSHX formula showed no effect in ECM protection and chondrocyte characteristic maintenance (Figures $4(\mathrm{e})$ and $4(\mathrm{f})$ ). The protein expression of pSMAD2/3 was promoted by BSHX formula-mediated serum but downregulated by $\operatorname{Tgf} \beta \mathrm{r} 2$ siRNA. However, after Tgf $\beta \mathrm{r} 2$ siRNA transfection, BSHX formula-mediated serum could not increase pSMAD2/3 expression (Figure $4(\mathrm{~g})$ ). The results insisted that BSHX formula exerts its cartilage regeneration promoting effect through TGF- $\beta$ pathway.

\section{Discussion}

In the present study, experiments were performed to elucidate the impact of BSHX formula on articular cartilage repair in full-thickness cartilage defect rat model. Relative gross appearance and histological and immunohistochemistry examination revealed prominently articular cartilage repair promoting effect on cartilage lesion. We also found BSHX formula-mediated serum can accelerate chondrocyte proliferation and ECM synthesis meanwhile can prevent matrix degradation and chondrocyte hypertrophy on chondrogenic
ATDC5 cells. However, the abovementioned biological role of BSHX formula was almost eliminated by downregulation of TGF- $\beta$ signaling.

Repairing of articular cartilage lesion remains a challenging issue because of its avascular and aneural nature [26]. All reparative approaches were trying but failed to achieve the ultimate goal of regeneration and restoration of the hyaline cartilage in the defected area [27]. Cartilage-to-cartilage integration is extremely difficult to achieve because cartilage displays low metabolism and contains dense, antiadhesive ECM [28]. Without reasonable intervention, the newly formed tissue always presents fibrocartilage phenotype with inadequate biomechanical property compared to hyaline cartilage. As we saw in model group at $8 \mathrm{w}$ (Figure 1(a)), mismatches in this property result in strain disparities between neocartilage and surrounding tissue, leading to tissue degradation [10]. Therefore, it is urgent for clinicians and researchers to find a promising way to deal with this disorder.

Chinese medicine acting as a nonsurgical treatment is easy to perform and less invasive. Recently, these natural products have taken more attention in biomedical applications. Ample evidence suggests TCM as being effective in articular cartilage protection. For example, Zhang et al. 

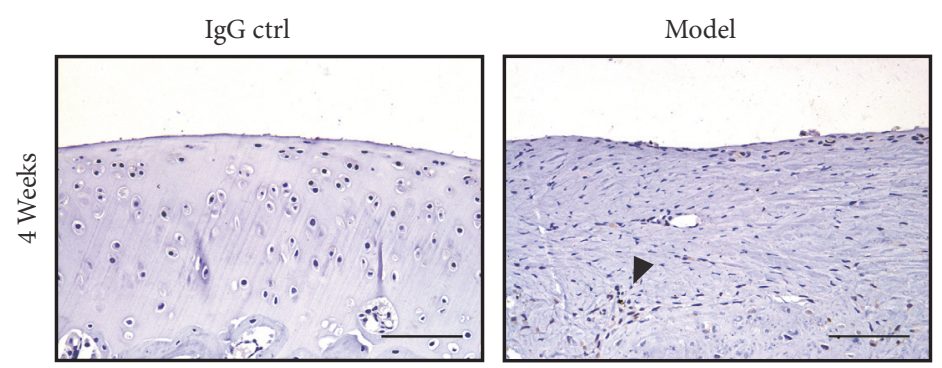

(a)

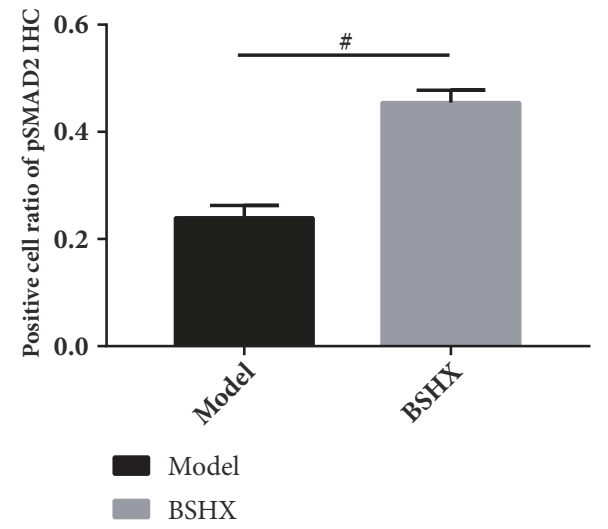

(b)

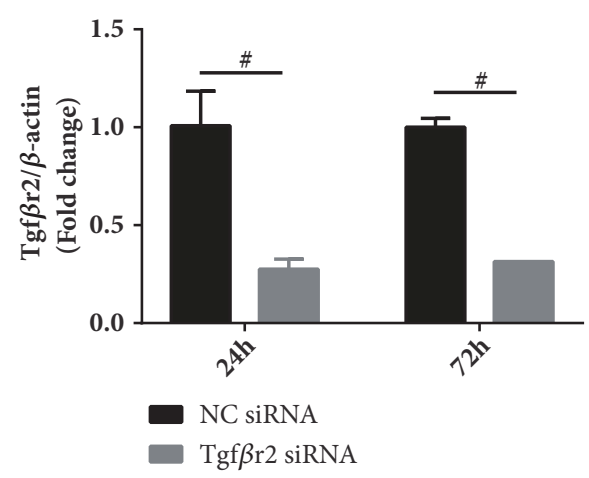

(c)

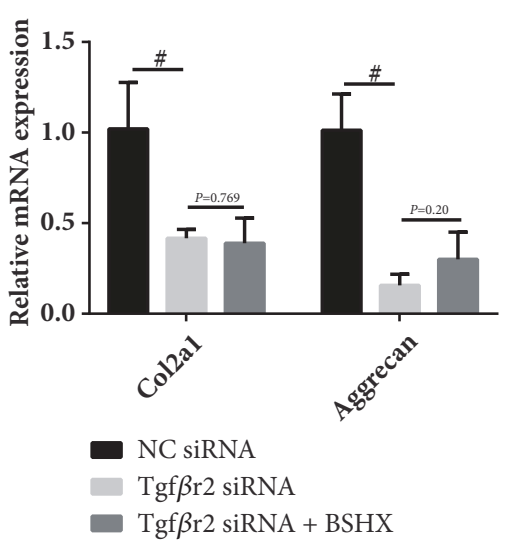

(d)

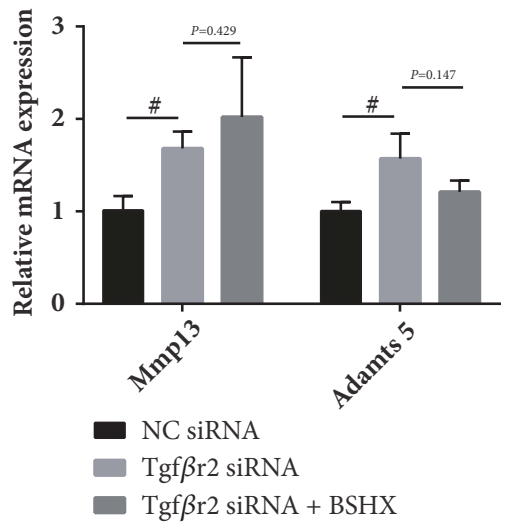

(e)

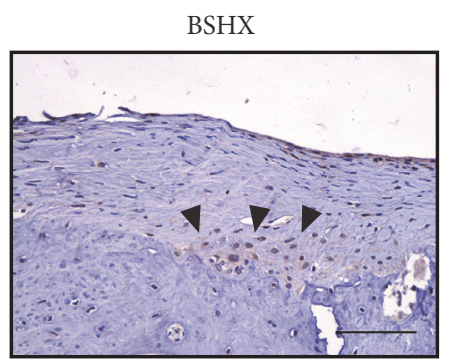
. 
researches and extends the benefits of TCM to articular cartilage repair.

Full-thickness cartilage defect often implicates subchondral bone, and latent bone marrow mesenchymal stromal cells (BMSCs) and local growth factors may participate in the regeneration process through subchondral bone bleeding [32]. Due to BMSC pluripotency, subchondral bone regenerates and remodels up to a critical size through endochondral ossification which is consistent to our finding in model group at $4 \mathrm{w}$ (Figure 2(a)). BMSC can also be differentiated into chondrocyte, but unlike bone, articular cartilage regeneration relies on sufficient chondrocytes and cartilage-specific ECM deposition which could not be achieved by BMSC alone [33]. Chondrocytes occupy only $2 \%$ of the total tissue volume of the articular cartilage whereas ECM and water account for the rest [34]. Col2 and Aggrecan, two major solid components of ECM, accumulated at early stage of cartilage formation and remodeling due to its maturation [35]. The change in Col10 and Runx2 expression that was associated with chondrocytes terminal differentiation may alter the phenotypic state of chondrocytes [36, 37]; combined with increasing expression of several proteinases such as Mmp13 and Adamts5, it could degrade Col2 and Aggrecan [38, 39] and finally lead to degeneration of neocartilage tissue. Our results showed BSHX formula could provide material basis of cartilage repair by promoting chondrocytes proliferation and ECM assembly. Furthermore, it can also prevent the neocartilage from degradation by decreasing matrix degrading enzyme and chondrocytes hypertrophy factors.

In this study, significant articular cartilage repair promoting effect was found in cartilage defect rats treated with BSHX formula. Our data showed that BSHX formula could elevate protein expressional level of Col2 and pSMAD2 and decrease Mmp13 and Runx2 in cartilage defect rat. In treating chondrogenic ATDC5 cells, we also found that BSHX formula could promote cell proliferation and prevent it from hypertrophy and increase ECM synthesis meanwhile preventing it from degradation. To further elucidate the mechanism of BSHX formula articular cartilage repairing effects, we investigated the efficiency of BSHX formula in $\operatorname{Tgf} \beta \mathrm{r} 2$ siRNA induced TGF- $\beta$ signaling downregulated chondrogenic ATDC5 cells. As reported in previous researches, TGF- $\beta$ signaling promotes chondrocytes to express ECM molecules, Col2, and Aggrecan to form cartilage tissue [40]. Deletion of Tgf $\beta \mathrm{r} 2$ resulted in upregulation of Runx2, Col10, Mmp13, and Adamts5 expression in chondrocytes [41]. In our study, the alteration of ECM deposition, chondrocyte hypertrophy-related genes, and $\mathrm{pSMAD} 2 / 3$ protein level observed in $\operatorname{Tgf} \beta \mathrm{r} 2$ downregulated chondrogenic ATDC5 cells could not be adjusted by BSHX formula, indicating BSHX formula exerts its articular cartilage repairing effects through TGF- $\beta$ signaling. However, Tgf $\beta$ r 2 siRNA could not completely impede the alteration of Adamts 5 and Aggrecan expression induced by BSHX formula. One possible reason for this finding is that about $70 \%$ of $\operatorname{Tgf} \beta \mathrm{r} 2$ gene was blocked in chondrogenic ADTC5 cells by Tgf $\beta \mathrm{r} 2$ siRNA; the transfection efficiency is close to the previous study [42]. $30 \%$ of $\operatorname{Tgf} \beta \mathrm{r} 2$ remains normal in chondrocytes. Besides, considering the multitarget of TCM, BSHX formula might also regulate the expression of these genes through other signaling pathways which need further investigation.

\section{Conclusion}

In current study, we found that BSHX formula promotes articular cartilage repair as a natural TGF- $\beta$ signaling activator via numerous ways, including acceleration of chondrocyte proliferation and maintaining chondrocyte phenotype, upregulates ECM accumulation, and inhibits matrix degradation either in or ex vivo. These findings enlighten us that TCM may be considered as an effective complementary and alternative medicine to cartilage damage; meanwhile, it encourages further research for this treatment.

\section{Data Availability}

The data used to support the findings of this study are available from the corresponding author upon request.

\section{Conflicts of Interest}

The authors declare no conflicts of interest regarding the publication of this paper.

\section{Authors' Contributions}

Rui Dong, Jun Ying, Taotao Xu, and Songfeng Hu performed the experiments. Peng Zhang, Chenjie Xia, and Liang Fang performed data analysis. Rui Dong and Jun Ying drafted the article. Hongting Jin and Pinger Wang were responsible for the conception and design of the study, revision of the paper, and the final approval of the version to be published. Rui Dong and Jun Ying contributed equally to this work.

\section{Acknowledgments}

This work was supported by the National Natural Science Foundation of China (Grant nos. 81774332, 81774346, 81873324, and 81873325). This research has been partially supported by Zhejiang grants funded by Provincial Natural Science Foundation of China (Grant nos. LY16H270010, LQ16H270007), the State Administration of Traditional Chinese Medicine of Zhejiang Province (Grant nos. 2016ZA048, 2018ZZ011, and 2018ZA034), the Project of Zhejiang Chinese Medical University (Grant no. 2C01801-01), and the Science and Technology Bureau of Shaoxing \& Health and Family Planning Commission of Shaoxing (Grant no. 2016QN006).

\section{References}

[1] D. Correa and S. A. Lietman, "Articular cartilage repair: current needs, methods and research directions," Seminars in Cell \& Developmental Biology, vol. 62, pp. 67-77, 2017.

[2] Z. Jia, Q. Liu, Y. Liang et al., "Repair of articular cartilage defects with intra-articular injection of autologous rabbit synovial fluid-derived mesenchymal stem cells," Journal of Translational Medicine, vol. 16, no. 1, 2018. 
[3] D. M. Findlay and J. S. Kuliwaba, "Bone-cartilage crosstalk: A conversation for understanding osteoarthritis," Bone Research, vol. 4, p. 16028, 2016.

[4] M. Brittberg, "Cell carriers as the next generation of cell therapy for cartilage repair: A review of the matrix-induced autologous chondrocyte implantation procedure," The American Journal of Sports Medicine, vol. 38, no. 6, pp. 1259-1271, 2010.

[5] D. C. Flanigan, J. D. Harris, T. Q. Trinh, R. A. Siston, and R. H. Brophy, "Prevalence of chondral defects in Athletes' Knees: a systematic review," Medicine \& Science in Sports \& Exercise, vol. 42, no. 10, pp. 1795-1801, 2010.

[6] N.-J. Chang, Y.-T. Lin, C.-C. Lin, H.-C. Wang, H.-C. Hsu, and M.-L. Yeh, “The repair of full-thickness articular cartilage defect using intra-articular administration of $\mathrm{N}$-acetyl-d-glucosamine in the rabbit knee: Randomized controlled trial," Biomedical Engineering Online, vol. 14, no. 1, article no. 105, 2015.

[7] S. A. Lietman, S. Miyamoto, P. R. Brown, N. Inoue, and A. H. Reddi, "The temporal sequence of spontaneous repair of osteochondral defects in the knees of rabbits is dependent on the geometry of the defect," The Journal of Bone \& Joint Surgery (British Volume), vol. 84, no. 4, pp. 600-606, 2002.

[8] Á. Berta, Z. Duska, F. Tóth, and L. Hangody, "Clinical experiences with cartilage repair techniques: Outcomes, indications, contraindications and rehabilitation," Eklem Hastalıkları ve Cerrahisi, vol. 26, no. 2, pp. 84-96, 2015.

[9] K. Mithoefer, T. Mcadams, R. J. Williams, P. C. Kreuz, and B. R. Mandelbaum, "Clinical efficacy of the microfracture technique for articular cartilage repair in the knee: an evidence-based systematic analysis," The American Journal of Sports Medicine, vol. 37, no. 10, pp. 2053-2063, 2009.

[10] D. J. Huey, J. C. Hu, and K. A. Athanasiou, "Unlike bone, cartilage regeneration remains elusive," Science, vol. 338, no. 6109, pp. 917-921, 2012.

[11] S. Imade, N. Kumahashi, S. Kuwata, J. Iwasa, and Y. Uchio, "Effectiveness and limitations of autologous osteochondral grafting for the treatment of articular cartilage defects in the knee," Knee Surgery, Sports Traumatology, Arthroscopy, vol. 20, no. 1, pp. 160-165, 2012.

[12] W. D. Bugbee, A. L. Pallante-Kichura, S. Görtz, D. Amiel, and R. Sah, "Osteochondral allograft transplantation in cartilage repair: Graft storage paradigm, translational models, and clinical applications," Journal of Orthopaedic Research, vol. 34, no. 1, pp. 31-38, 2016.

[13] B. Johnstone, M. Alini, and M. Cucchiarini, "Tissue engineering for articular cartilage repair-the state of the art," European cells materials, vol. 25, pp. 248-267, 2013.

[14] F. Zhu, L. Yin, L. Ji et al., "Suppressive effect of Sanmiao formula on experimental gouty arthritis by inhibiting cartilage matrix degradation: An In Vivo and In Vitro Study," International Immunopharmacology, vol. 30, pp. 36-42, 2016.

[15] F. Wang, L. Shi, Y. Zhang et al., "A Traditional Herbal Formula Xianlinggubao for Pain Control and Function Improvement in Patients with Knee and Hand Osteoarthritis: A Multicenter, Randomized, Open-Label, Controlled Trial," Evidence-Based Complementary and Alternative Medicine, vol. 2018, Article ID 1827528, 10 pages, 2018.

[16] L. Li, H. Liu, W. Shi et al., "Insights into the action mechanisms of traditional chinese medicine in osteoarthritis," EvidenceBased Complementary and Alternative Medicine, vol. 2017, Article ID 5190986, 13 pages, 2017.

[17] H. Wang, Z. Shen, and J. Zeng, "Clinical observation of nourishing kidney and activating blood therapy for degenerative osteoarthritis," Journal of New Chinese Medicine, vol. 49, no. 4, pp. 65-67, 2017.

[18] J. Yuan, C. Luo, and Y. Huang, "Clinical study on oral application of self-made Bushen Huoxue Tang for treatment of early knee osteoarthritis," The Journal of Traditional Chinese Orthopedics and Traumatology, vol. 29, no. 08, pp. 26-32, 2017.

[19] P. E. Wang, L. Zhang, and J. Ying, "Bushenhuoxue formula attenuates cartilage degeneration in an osteoarthritic mouse model through TGF-beta/MMP13 signaling," Journal of Translational Medicine, vol. 16, no. 1, p. 72, 2018.

[20] J. Shen, S. Li, and D. Chen, "TGF- $\beta$ signaling and the development of osteoarthritis," Bone Research, vol. 2, Article ID 14002, 2014.

[21] J. Ying, P. Wang, S. Zhang et al., "Transforming growth factorbetal promotes articular cartilage repair through canonical Smad and Hippo pathways in bone mesenchymal stem cells," Life Sciences, vol. 192, pp. 84-90, 2018.

[22] T. Zhou, X. Li, G. Li et al., "Injectable and thermosensitive TGF$\beta 1$-loaded PCEC hydrogel system for in vivo cartilage repair," Scientific Reports, vol. 7, no. 1, 2017.

[23] S. Y. Xu, R. L. Bian, X. Chen et al., "Dose problems in pharmacological experiments," in Experimental Methodology of Pharmacology, R. Y. Sun, Y. M. Ma, and Z. Y. Hong, Eds., pp. 202-203, People's Medical Publishing House, Beijing, China, 3rd edition, 2002.

[24] M. Brittberg and C. S. Winalski, "Evaluation of cartilage injuries and repair," The Journal of Bone \& Joint Surgery, vol. 85, supplement 2, pp. 58-69, 2003.

[25] S. Isaka, A. Someya, S. Nakamura et al., "Evaluation of the effect of oral administration of collagen peptides on an experimental rat osteoarthritis model," Experimental and Therapeutic Medicine, vol. 13, no. 6, pp. 2699-2706, 2017.

[26] I. M. Khan, S. J. Gilbert, S. K. Singhrao, V. C. Duance, and C. W. Archer, "Cartilage integration: Evaluation of the reasons for failure of integration during cartilage repair. A review," European Cells and Materials, vol. 16, pp. 26-39, 2008.

[27] Y. A. Rim, Y. Nam, N. Park, J. Lee, S. Park, and J. H. Ju, "Repair potential of nonsurgically delivered induced pluripotent stem cell-derived chondrocytes in a rat osteochondral defect model," Journal of Tissue Engineering and Regenerative Medicine, vol. 12, no. 8, pp. 1843-1855, 2018.

[28] J. van de Breevaart Bravenboer, C. D. In der Maur, P. K. Bos et al., "Improved cartilage integration and interfacial strength after enzymatic treatment in a cartilage transplantation model.," Arthritis research \& therapy, vol. 6, no. 5, pp. R469-476, 2004.

[29] L. Zhang, P. Wang, J. Ying et al., "Yougui Pills Attenuate Cartilage Degeneration via Activation of TGF- $\beta$ /Smad Signaling in Chondrocyte of Osteoarthritic Mouse Model," Frontiers in Pharmacology, vol. 8, p. 611, 2017.

[30] K. Wang, D. Zhang, Y. Liu et al., “Traditional Chinese medicine formula Bi-Qi capsule alleviates rheumatoid arthritis-induced inflammation, synovial hyperplasia, and cartilage destruction in rats," Arthritis research therapy, vol. 20, no. 1, p. 43, 2018.

[31] X. Wang, P. Shen, D. Tang et al., "Effects of Qi-Fang-XiBi-Granules on cartilage morphology and C/ebp $\alpha$ promoter methylation in rats with knee osteoarthritis," Evidence-Based Complementary and Alternative Medicine, vol. 2018, Article ID 2074976, 12 pages, 2018.

[32] M. F. Sommerfeldt, R. A. Magnussen, T. E. Hewett, C. C. Kaeding, and D. C. Flanigan, "Microfracture of Articular Cartilage," JBJS Reviews, vol. 4, no. 6, p. 1, 2016. 
[33] M. Nishimori, M. Deie, A. Kanaya, H. Exham, N. Adachi, and M. Ochi, "Repair of chronic osteochondral defects in the rat," The Journal of Bone \& Joint Surgery (British Volume), vol. 88, no. 9, pp. 1236-1244, 2006.

[34] M. Ulrich-Vinther, M. D. Maloney, E. M. Schwarz, R. Rosier, and R. J. O'Keefe, "Articular cartilage biology.," Journal of the American Academy of Orthopaedic Surgeons, vol. 11, no. 6, pp. 421-430, 2003.

[35] S. Camarero-Espinosa, B. Rothen-Rutishauser, E. J. Foster, and C. Weder, "Articular cartilage: From formation to tissue engineering," Biomaterials Science, vol. 4, no. 5, pp. 734-767, 2016.

[36] P. M. van der Kraan and W. B. van den Berg, "Chondrocyte hypertrophy and osteoarthritis: role in initiation and progression of cartilage degeneration?" Osteoarthritis and Cartilage, vol. 20, no. 3, pp. 223-232, 2012.

[37] M. M.-G. Sun and F. Beier, "Chondrocyte hypertrophy in skeletal development, growth, and disease," Birth Defects Research Part C-Embryo Today: Reviews, vol. 102, no. 1, pp. 74-82, 2014.

[38] L. A. Neuhold, L. Killar, W. Zhao et al., "Postnatal expression in hyaline cartilage of constitutively active human collagenase3 (MMP-13) induces osteoarthritis in mice," The Journal of Clinical Investigation, vol. 107, no. 1, pp. 35-44, 2001.

[39] H. Stanton, F. M. Rogerson, C. J. East et al., "ADAMTS5 is the major aggrecanase in mouse cartilage in vivo and in vitro," Nature, vol. 434, no. 7033, pp. 648-652, 2005.

[40] P. Xia, X. Wang, Y. Qu et al., "TGF-betal-induced chondrogenesis of bone marrow mesenchymal stem cells is promoted by low-intensity pulsed ultrasound through the integrin-mTOR signaling pathway," Stem Cell Research Therapy, vol. 8, no. 1, p. 281, 2017.

[41] J. Shen, J. Li, B. Wang et al., "Deletion of the transforming growth factor $\beta$ Receptor type II gene in articular chondrocytes leads to a progressive osteoarthritis-like phenotype in mice," Arthritis \& Rheumatology, vol. 65, no. 12, pp. 3107-3119, 2013.

[42] Z. S. Hashemi, M. F. Moghadam, and M. Soleimani, "Comparison of TGFbR2 down-regulation in expanded HSCs on MBA/ DBM scaffolds coated by UCB stromal cells," In Vitro Cellular \& Developmental Biology - Animal, vol. 51, no. 5, pp. 495-506, 2015. 


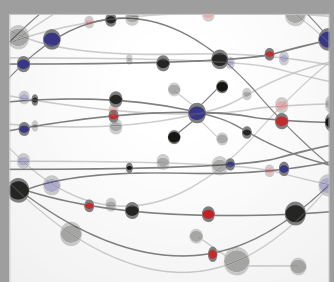

The Scientific World Journal
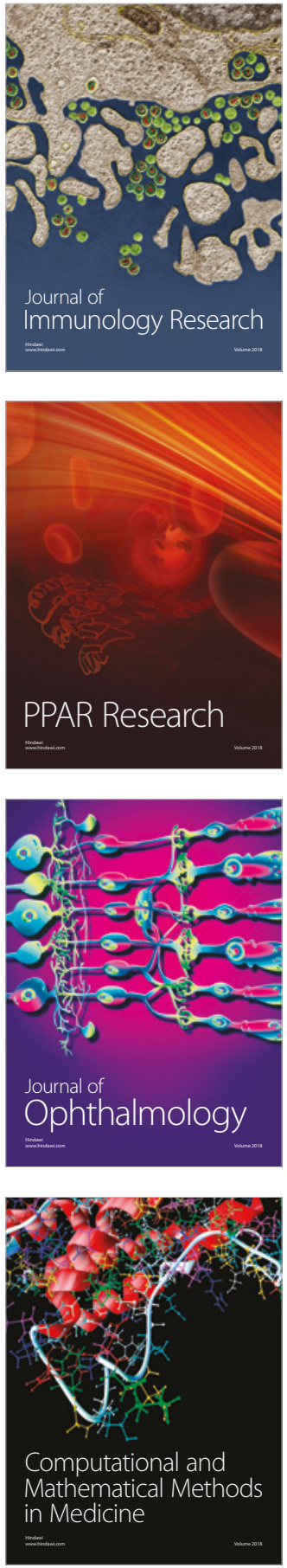

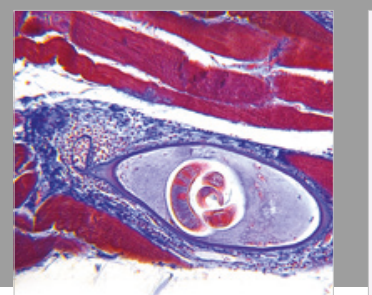

Gastroenterology Research and Practice

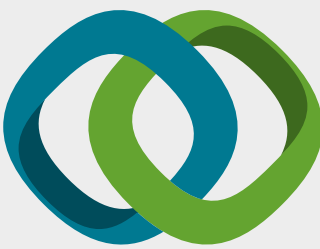

\section{Hindawi}

Submit your manuscripts at

www.hindawi.com
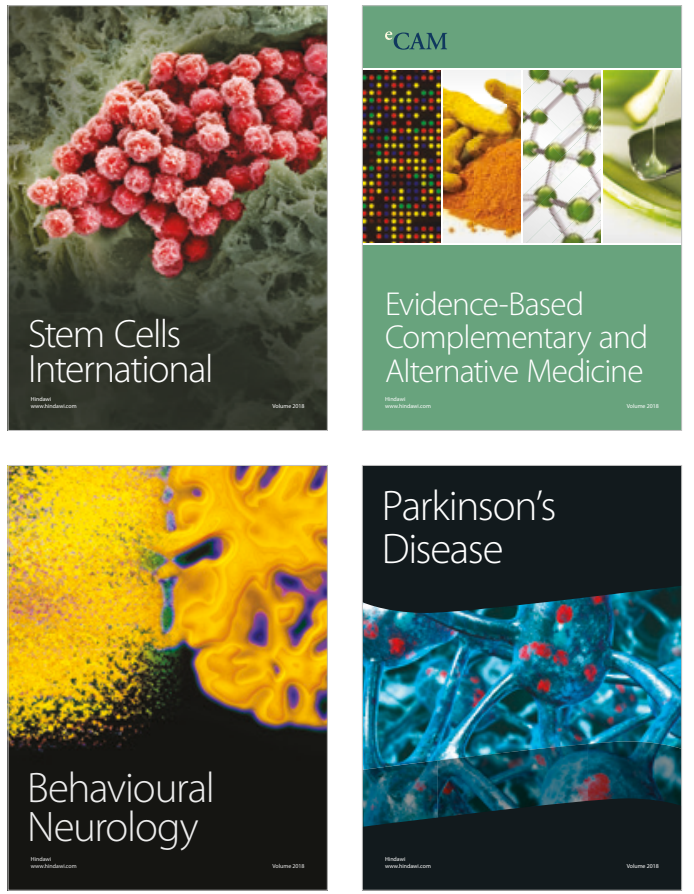

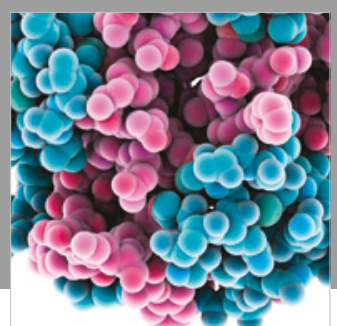

ournal of

Diabetes Research

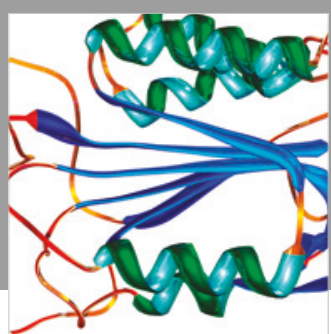

Disease Markers
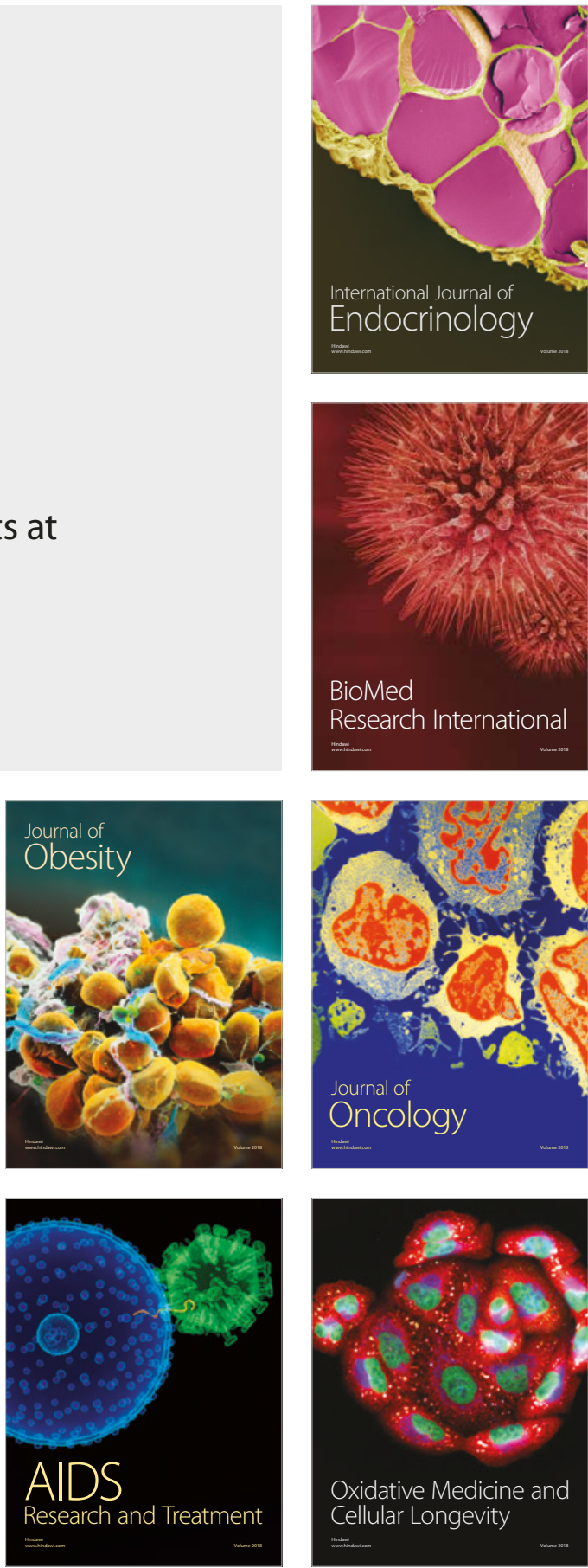